$10-1-2008$

\title{
The Matrices R and G of Matrix Analytic Methods and The Time- Inhomogeneous Periodic Quasi-Birth-and-Death Process
}

Barbara H. Margolius

Cleveland State University, b.margolius@csuohio.edu

Follow this and additional works at: https://engagedscholarship.csuohio.edu/scimath_facpub

Part of the Mathematics Commons

How does access to this work benefit you? Let us know!

Publisher's Statement

The final publication is available at Springer via http://dx.doi.org/10.1007/s11134-008-9090-9

\section{Repository Citation}

Margolius, Barbara H., "The Matrices R and G of Matrix Analytic Methods and The Time-Inhomogeneous Periodic Quasi-Birth-and-Death Process" (2008). Mathematics Faculty Publications. 238.

https://engagedscholarship.csuohio.edu/scimath_facpub/238

This Article is brought to you for free and open access by the Mathematics and Statistics Department at EngagedScholarship@CSU. It has been accepted for inclusion in Mathematics Faculty Publications by an authorized administrator of EngagedScholarship@CSU. For more information, please contact library.es@csuohio.edu. 


\title{
The matrices $R$ and $G$ of matrix analytic methods and the time-inhomogeneous periodic Quasi-Birth-and-Death process
}

\author{
B.H. Margolius
}

\begin{abstract}
We solve for the asymptotic periodic distribution of the continuous time quasi-birth-and-death process with time-varying periodic rates in terms of $\hat{\mathbf{R}}$ and $\hat{\mathbf{G}}$ matrix functions which are analogues of the $\mathbf{R}$ and $\mathbf{G}$ matrices of matrix analytic methods. We evaluate these QBDs numerically by solving for $\hat{\mathbf{R}}$ numerically.
\end{abstract}

Mathematics Subject Classification (2000) 60K25 - 60J80 $\cdot 60 \mathrm{~J} 10$

\section{Introduction}

Queues with time-varying rates have been considered in the literature at least since Kolmogorov considered the waiting problem in a paper in 1931 [14, 15]. His approach was to set up the Chapman-Kolmogorov equations for the system. Gnedenko and Kovalenko provide a brief treatment of time-varying customer streams [10, pp. 76-83] in their Introduction to Queueing Theory. Markovian single and multiple server queues with time-varying parameters have been analyzed by Leese and Boyd [19], Zhang and Coyle [29] and Margolius [22-24]. Recently, Alfa and Margolius [2] have analyzed discrete time systems with time-varying periodic transition probabilities using matrix analytic methods.

Motivating applications for queues with time-varying parameters include airport congestion (Koopman [17], Daniel [5] and Peterson, Bertsimas and Odoni [27]), police calls for service, call centers [16], streaming and data traffic in a multiserver network (Delcoigne et al. [6]), traffic congestion, and many others. 
In this paper, we focus on systems with time-varying periodic parameters. Afanas'eva [3], Breuer [4], Koopman [17], Harrison and Lemoine [13] and others have written about existence conditions for an asymptotic periodic solution when parameters are periodic. Zeifman et al. [28] have studied convergence of the transient solution to the asymptotic solution for queues with time-varying periodic parameters. Additional references are available from these sources. Green and Kolesar [11, 12] study approximation methods for queues with time-varying parameters motivated initially by the police dispatching problem. Avi Mandelbaum [7, 16, 20,21] and others have written a series of papers related to queues with time-varying parameters. Call centers are a primary motivation in several of the papers. A survey of the call center literature based on mathematical queueing theory appears in [16].

In this paper, we consider quasi-birth-and-death processes with time-varying periodic rates. This extends results in [25] and puts the time-varying periodic QBD in the context of matrix analytic methods $[18,26]$. The approach requires the numerical solution of an integral equation over one time period.

We consider a Markov chain $\left\{\left(X_{t}, J_{t}\right), t \geq 0\right\}$ on the two-dimensional state space $\{(n, i): n \geq 0,1 \leq i \leq m\}$. The first coordinate $n$ is called the level, and the second coordinate $j$ is called the phase of the state $(n, j)$. We shall also use the word level to denote the whole subset $\ell(n)=\{(n, 1),(n, 2), \ldots,(n, m)\}$. The number $m$ of states in each level is finite. We assume throughout this paper that the process is irreducible, so that there exists a path between any two states.

The Markov chain is called a QBD if one-step transitions from a state are restricted to states in the same level or in the two adjacent levels: it is possible to move in one step from $(n, j)$ to $\left(n^{\prime}, j^{\prime}\right)$ only if $n^{\prime}=n, n+1$ or $n-1$ (provided in the last case that $n \geq 1)$.

The transition rates are assumed to be level-independent. Thus the infinitesimal generator is block tri-diagonal and has the following form:

$$
\mathbf{Q}(t)=\left[\begin{array}{ccccc}
\mathbf{B}(t) & \mathbf{A}_{1}(t) & 0 & 0 & \cdots \\
\mathbf{A}_{-1}(t) & \mathbf{A}_{0}(t) & \mathbf{A}_{1}(t) & 0 & \cdots \\
0 & \mathbf{A}_{-1}(t) & \mathbf{A}_{0}(t) & \mathbf{A}_{1}(t) & \cdots \\
0 & 0 & \mathbf{A}_{-1}(t) & \mathbf{A}_{0}(t) & \cdots \\
\vdots & \vdots & \vdots & \vdots & \ddots
\end{array}\right]
$$

where $\mathbf{A}_{-1}(t), \mathbf{A}_{0}(t), \mathbf{A}_{1}(t)$ and $\mathbf{B}(t)$ are square matrices of order $m$. We chose this notation rather than the alternative notation for QBDs in which the $\mathbf{A}_{i}$ matrices are numbered in descending order. The purpose of the notational scheme used in this paper is to link the subscript to transitions among levels, so that, for example, the $(i, j)$ component of the matrix $\mathbf{A}_{-1}(t)$ gives the rate at time $t$ at which transitions from phase $i$ to phase $j$ and down one level occur.

In this paper, we focus on the asymptotic periodic solution to the QBD. We temporarily assume that the QBD is the solution of the system $\dot{\boldsymbol{\pi}}(t)=\boldsymbol{\pi}(t) \mathbf{Q}(t)$, $\boldsymbol{\pi}(t) \mathbf{1}=1$. The asymptotic periodic distribution will satisfy the condition $\boldsymbol{\pi}(s)=$ $\pi(s+n T)$ where $T$ is the period, and $n$ is any integer. In what follows, we assume that the period is of length 1 so our boundary condition becomes $\pi(s)=\pi(s+n)$.

For each time $s$ within the period, $\pi(s)$ is the solution to an embedded discrete time Markov chain. However, we do not have the transition probability matrix for 
this chain available to us and if we did, computing the stationary distribution for the chain would be challenging because the matrix would be dense, though entries distant from the diagonal would be negligible. What we have available is the time-dependent rate matrix.

We partition $\pi(t)$ by levels into subvectors $\pi_{n}(t), n \geq 0$, where $\pi_{n}(t)$ has $m$ components. The defining system satisfies the Chapman-Kolmogorov forward equations

$$
\begin{aligned}
& \dot{\boldsymbol{\pi}}_{0}(t)=\boldsymbol{\pi}_{0}(t) \mathbf{B}(t)+\boldsymbol{\pi}_{1}(t) \mathbf{A}_{-1}(t) \\
& \dot{\boldsymbol{\pi}}_{n}(t)=\boldsymbol{\pi}_{n-1}(t) \mathbf{A}_{1}(t)+\boldsymbol{\pi}_{n}(t) \mathbf{A}_{0}(t)+\boldsymbol{\pi}_{n+1}(t) \mathbf{A}_{-1}(t)
\end{aligned}
$$

with the additional requirement that

$$
\sum_{n=0}^{\infty} \pi_{n}(t) \mathbf{1}=1
$$

\section{Random walk}

We associate a generalized random walk to the QBD [25]. Consider the Markov chain $\left\{\left(L_{t}, \zeta_{t}\right), t \geq s\right\}$ on the state space $\mathbb{Z} \times\{1,2, \ldots, m\}$ with transition intensities depending only on the second coordinate and the time $t$. The Chapman-Kolmogorov forward equation for this generalized random walk is given by

$$
\dot{\mathbf{p}}_{n}(t)=\mathbf{p}_{n-1}(t) \mathbf{A}_{1}(t)+\mathbf{p}_{n}(t) \mathbf{A}_{0}(t)+\mathbf{p}_{n+1}(t) \mathbf{A}_{-1}(t)
$$

for all $n \in \mathbb{Z}$. Define $\left[\phi_{n}(s, t)\right]_{i j}=P\left\{L_{t}=n+k, \zeta_{t}=j \mid L_{s}=k, \zeta_{s}=i\right\}$ as the probability that the random walk traveled $n$ more levels to the right than to the left during the time interval $(s, t)$ and changed from phase $i$ to phase $j$. For example, when the $\mathbf{A}_{l}(t), l=-1,0,1$, are scalar with

$$
\begin{aligned}
\dot{p}_{n}(t)= & \lambda(t) p_{n-1}(t)-(\lambda(t)+\mu(t)) p_{n}(t)+\mu(t) p_{n+1}(t) \\
\phi_{n}(s, t)= & \left(\frac{\int_{s}^{t} \lambda(u) d u}{\int_{s}^{t} \mu(u) d u}\right)^{n / 2} \\
& \times I_{n}\left(2 \sqrt{\int_{s}^{t} \lambda(u) d u \int_{s}^{t} \mu(u) d u}\right) e^{-\int_{s}^{t} \mu(u) d u-\int_{s}^{t} \lambda(u) d u}
\end{aligned}
$$

where $I_{n}(\cdot)$ is the $n$th modified Bessel function [23].

To see this, consider a Poisson process $X(t)$. If $X(t)$ is a homogeneous Poisson process with rate $\lambda$,

$$
\operatorname{Pr}\{X(t)=j+k \mid X(s)=k\}=\frac{(\lambda(t-s))^{j}}{j !} e^{-\lambda(t-s)},
$$

and for an inhomogeneous process with rate $\lambda(t)$,

$$
\operatorname{Pr}\{X(t)=j+k \mid X(s)=k\}=\frac{\left(\int_{s}^{t} \lambda(\nu) d \nu\right)^{j}}{j !} e^{-\int_{s}^{t} \lambda(v) d v} .
$$


For an inhomogeneous random walk $Z(t)$, with jumps to the right occurring according to the process $X(t)$ at rate $\lambda(t)$ and jumps to the left occurring according to the inhomogeneous process $Y(t)$ at rate $\mu(t)$,

$$
\begin{aligned}
\operatorname{Pr}\{Z(t)=n \mid Z(s)=0\} & =\sum_{j=0}^{\infty} \operatorname{Pr}\{X(t)=n+j\} \operatorname{Pr}\{Y(t)=j\} \\
& =e^{-\int_{s}^{t}(\lambda(\nu)+\mu(v)) d v} \sum_{j=0}^{\infty} \frac{\left(\int_{s}^{t} \lambda(\nu) d \nu\right)^{n+j}\left(\int_{s}^{t} \mu(\nu) d \nu\right)^{j}}{(n+j) ! j !}
\end{aligned}
$$

for $n \geq 0$. The expression is similar for $n<0$. This is $\phi_{n}(s, t)$ from (2).

For an $E_{m}(t) / M(t) / 1$ queue [25],

$$
\begin{aligned}
\mathbf{A}_{1}(t)= & {\left[\begin{array}{cccc}
\cdot & \cdots & \cdot & v(t) \\
\cdot & \cdots & \cdots & \cdot \\
\vdots & \vdots & \vdots & \vdots \\
\cdot & \cdots & \cdots & \cdot
\end{array}\right], } \\
\mathbf{A}_{0}(t)= & {\left[\begin{array}{cccc}
-v(t)-\mu(t) & \cdot & \cdot & \cdot \\
v(t) & -v(t)-\mu(t) & \cdot & \cdot \\
\cdot & \ddots & \ddots & \cdot \\
\cdot & \cdot & v(t) & -v(t)-\mu(t)
\end{array}\right], } \\
\mathbf{A}_{-1}(t)= & {\left[\begin{array}{ccc}
\mu(t) & \cdot & \cdot \\
\cdot & \ddots & \cdot \\
\cdot & \cdot & \mu(t)
\end{array}\right], }
\end{aligned}
$$

where $v(t)$ is the rate at which a transition occurs from one phase of the arrival process to the next. Arrivals have an Erlang $(m, v(t))$ distribution where the transition rate $v(t)$ depends on time within the period for the QBD $\left(X_{t}, J_{t}\right)$. The service distribution is exponential with time-varying rate $\mu(t)$. The generalized random walk transition probabilities are then given by the Toeplitz matrix

$$
\begin{aligned}
\phi_{n}(s, t)= & e^{-\int_{s}^{t}(\mu(u)+v(u)) d u} \\
& \times\left[\begin{array}{cccc}
g^{(0)}(n, s, t) & g^{(-1)}(n, s, t) & \ldots & g^{(-j+1)}(n, s, t) \\
g^{(1)}(n, s, t) & g^{(0)}(n, s, t) & \ldots & g^{(-j+2)}(n, s, t) \\
\vdots & \ddots & \ddots & \vdots \\
g^{(j-1)}(n, s, t) & g^{(j-2)}(n, s, t) & \ldots & g^{(0)}(n, s, t)
\end{array}\right]
\end{aligned}
$$

where

$$
g^{(l)}(n, s, t)=\sum_{m=0 \vee-k}^{\infty} \frac{\left(\int_{s}^{t} v(u) d u\right)^{j(m+n)+l}\left(\int_{s}^{t} \mu(u) d u\right)^{m}}{(j(m+n)+l) ! m !}
$$


for $j>l \geq 0$, and

$$
g^{(l)}(n, s, t)=\sum_{m=0 \vee 1-l}^{\infty} \frac{\left(\int_{s}^{t} \nu(u) d u\right)^{j(m+n)-l}\left(\int_{s}^{t} \mu(u) d u\right)^{m}}{(j(m+n)-l) ! m !}
$$

for $-j<l<0$.

For the $M(t) / E_{m}(t) / 1$ queue, the form of $\phi_{n}(s, t)$ is similar:

$$
\begin{aligned}
& \mathbf{A}_{-1}(t)=\left[\begin{array}{cccc} 
& \cdots & \cdot & v(t) \\
\cdot & \cdots & \cdots & \cdot \\
\vdots & \vdots & \vdots & \vdots \\
\cdot & \cdots & \cdots & \cdot
\end{array}\right] \\
& \mathbf{A}_{0}(t)=\left[\begin{array}{cccc}
-v(t)-\lambda(t) & \cdot & \cdot & \cdot \\
v(t) & -v(t)-\lambda(t) & \cdot & \cdot \\
\cdot & \ddots & \ddots & \cdot \\
\cdot & \cdot & v(t) & -v(t)-\lambda(t)
\end{array}\right], \\
& \mathbf{A}_{1}(t)=\left[\begin{array}{ccc}
\lambda(t) & \cdot & \cdot \\
\cdot & \ddots & \cdot \\
\cdot & \cdot & \lambda(t)
\end{array}\right],
\end{aligned}
$$

where $v(t)$ is the rate at which a transition occurs from one phase of the departure process to the next. Departures have an Erlang $(m, v(t))$ distribution where the transition rate depends on time within the period for the QBD $\left(X_{t}, J_{t}\right)$. The arrival distribution is Poisson with time-varying rate $\lambda(t)$. The generalized random walk transition probabilities are then given by the Toeplitz matrix

$$
\begin{aligned}
\phi_{n}(s, t)= & e^{-\int_{s}^{t}(\lambda(u)+v(u)) d u} \\
& \times\left[\begin{array}{cccc}
g^{(0)}(n, s, t) & g^{(-1)}(n, s, t) & \ldots & g^{(-m+1)}(n, s, t) \\
g^{(1)}(n, s, t) & g^{(0)}(n, s, t) & \ldots & g^{(-m+2)}(n, s, t) \\
\vdots & \ddots & \ddots & \vdots \\
g^{(j-1)}(n, s, t) & g^{(m-2)}(n, s, t) & \ldots & g^{(0)}(n, s, t)
\end{array}\right]
\end{aligned}
$$

where

$$
g^{(l)}(n, s, t)=\sum_{j=0 \vee-n}^{\infty} \frac{\left(\int_{s}^{t} v(u) d u\right)^{m j+l}\left(\int_{s}^{t} \lambda(u) d u\right)^{j+n}}{(m j+l) !(j+n) !}
$$

for $l \geq 0$, and

$$
g^{(l)}(n, s, t)=\sum_{j=0 \vee 1-l}^{\infty} \frac{\left(\int_{s}^{t} v(u) d u\right)^{m j-l}\left(\int_{s}^{t} \lambda(u) d u\right)^{j+n}}{(m j-l) !(j+n) !}
$$

for $l<0$. 
$\phi_{n}(s, t)$ is the coefficient on $z^{n}$ for the function $\Phi_{z}(s, t)$ which solves the differential equation

$$
\frac{\partial}{\partial t} \Phi_{z}(s, t)=\Phi_{z}(s, t)\left(z \mathbf{A}_{1}(t)+\mathbf{A}_{0}(t)+z^{-1} \mathbf{A}_{-1}(t)\right) .
$$

In most cases, an analytic expression for $\phi_{n}(s, t)$ will not be known. We discuss how to approximate these matrix functions in Sect. 6.

The $\phi_{n}(s, t)$ satisfy the system of differential equations (1), so

$$
\frac{\partial}{\partial t} \phi_{n}(s, t)=\phi_{n-1}(s, t) \mathbf{A}_{1}(t)+\phi_{n}(s, t) \mathbf{A}_{0}(t)+\phi_{n+1}(s, t) \mathbf{A}_{-1}(t)
$$

and

$$
\frac{\partial}{\partial s} \phi_{n}(s, t)=-\mathbf{A}_{1}(s) \phi_{n-1}(s, t)-\mathbf{A}_{0}(s) \phi_{n}(s, t)-\mathbf{A}_{-1}(s) \phi_{n+1}(s, t) .
$$

Furthermore, if $\mathbf{E}_{0}(s, t)$ is the solution of the evolution equation given by $\frac{\partial}{\partial t} \mathbf{E}_{0}(s, t)=$ $\mathbf{E}_{0}(s, t) \mathbf{A}_{0}(t)$ and $\frac{\partial}{\partial t} \mathbf{E}_{0}(s, t)=-\mathbf{A}_{0}(s) \mathbf{E}_{0}(s, t)$, then

$$
\frac{\partial}{\partial t}\left[\phi_{n}(s, t) \mathbf{E}_{0}(t, u)\right]=\phi_{n-1}(s, t) \mathbf{A}_{1}(t) \mathbf{E}_{0}(t, u)+\phi_{n+1}(s, t) \mathbf{A}_{-1}(t) \mathbf{E}_{0}(t, u)
$$

and

$$
\begin{aligned}
\int_{s}^{u} \frac{\partial}{\partial t}\left[\phi_{n}(s, t) \mathbf{E}_{0}(t, u)\right] d t= & \phi_{n}(s, u)-\phi_{n}(s, s) \mathbf{E}_{0}(s, u) \\
= & \int_{s}^{u}\left(\phi_{n-1}(s, t) \mathbf{A}_{1}(t) \mathbf{E}_{0}(t, u)\right. \\
& \left.+\phi_{n+1}(s, t) \mathbf{A}_{-1}(t) \mathbf{E}_{0}(t, u)\right) d u .
\end{aligned}
$$

$\phi_{n}(s, s)=\mathbf{0}$ for $n \neq 0$ and $\phi_{0}(s, s)=\mathbf{I}$ where $\mathbf{I}$ is the $m \times m$ identity matrix.

We need some additional definitions. For the functions $\phi_{n}(s, t), n \in \mathbb{Z}$, and any function $f(s, t)$ with $s \leq t$, define $\phi_{n} \star f(s, t)$ as

$$
\phi_{n} \star f(s, t)=\int_{s}^{t}\left(\phi_{n-1}(s, u) \mathbf{A}_{1}(u)+\phi_{n+1}(s, u) \mathbf{A}_{-1}(u)\right) f(u, t) d u .
$$

Define the functions $f_{n}(s, t)$ as solutions of the Volterra equations of the second kind:

$$
\begin{aligned}
f_{n}(s, t)= & \phi_{n-1}(s, t) \mathbf{A}_{1}(t)+\phi_{n+1}(s, t) \mathbf{A}_{-1}(t) \\
& -\int_{s}^{t} f_{n}(s, u)\left(\phi_{-1}(u, t) \mathbf{A}_{1}(t)+\phi_{1}(u, t) \mathbf{A}_{-1}(t)\right) d u .
\end{aligned}
$$

Define $\phi_{n} \star \phi_{0}^{\star-1} \star \phi_{m}(s, t)=\int_{s}^{t} f_{n}(s, u) \phi_{m}(u, t) d u$. Let $H_{m}=\inf \left\{\tau \mid L_{\tau}=m, \tau>s\right\}$ be the first hitting time after $s$ that the process enters $\ell(m)$. 
Lemma 1 For all integers $n$ and $m$, the quantity

$$
\begin{aligned}
& {\left[\phi_{n} \star \phi_{0}^{\star-1} \star \phi_{m}(s, t)\right]_{i j}} \\
& \quad=P\left\{L_{t}=n+m+k, \zeta_{t}=j, s<H_{n+k}<t \mid L_{s}=k, \zeta_{s}=i\right\}
\end{aligned}
$$

yields the probability that given the random walk was in phase $i$ and $\ell(k)$ at time $s$, that it hits $\ell(k+n)$ before time $t$, and it is in $\ell(n+m+k)$ and phase $j$ at time $t$.

Proof Refer to [22] (Lemma 3, p. 72) for a proof of this result where the $\mathbf{A}_{i}(t)$ are scalars. The matrix case is a direct generalization of the scalar case and the details are omitted.

Remark Note that if $|m|+|n|=|m+n|$, then $\phi_{n} \star \phi_{0}^{\star-1} \star \phi_{m}(s, t)=\phi_{m+n}(s, t)$ and, in particular, $\phi_{0} \star \phi_{0}^{\star-1} \star \phi_{m}(s, t)=\phi_{m}(s, t)$ and $\phi_{n} \star \phi_{0}^{\star-1} \star \phi_{0}(s, t)=\phi_{n}(s, t)$.

\section{Theorem 1 Let}

$$
[N(s, t)]_{i j}=P\left\{L_{t}=n, \zeta_{t}=j, L_{u} \neq n-1, s<u<t \mid L_{s}=n, \zeta_{s}=i\right\},
$$

then

$$
N(s, t)=\phi_{0}(s, t)-\phi_{-1} \star \phi_{0}^{\star(-1)} \star \phi_{1}(s, t) .
$$

Proof $N(s, t)$ is a matrix of transition probabilities for which transitions to any level lower than the initial level are taboo. These taboo transition probabilities for the QBD and the generalized random walk are the same for levels greater than zero. Recall that for the generalized random walk, $\phi_{0}(s, t)$ gives the probability of returning to or staying in the time $s$ level by time $t$ and remaining in that level until some time after $t$. Some random walks which return to the time $s$ level by time $t$ will have entered $\ell\left(L_{s}-1\right)$, a level lower than the time $s$ level. By Lemma 1 , the probability of a transition from phase $i$, level $n$ at time $s$ to phase $j$, level $n$ at time $t$ with a visit to level $n-1$ at some time $u, s<u<t$ is given by $\left[\phi_{-1} \star \phi_{0}^{\star(-1)} \star \phi_{1}(s, t)\right]_{i, j}$. Therefore, the matrix of taboo probabilities of not entering a lower level than the initial one and beginning and ending in the same level is given by $\phi_{0}(s, t)-\phi_{-1} \star \phi_{0}^{\star(-1)} \star \phi_{1}(s, t)$.

Theorem 2 Assume that the Markov process is irreducible. If an asymptotic periodic distribution exists, then it satisfies the relation

$$
\begin{aligned}
\boldsymbol{\pi}_{n+1}(t) & =\lim _{k \rightarrow \infty} \int_{s}^{t+k} \boldsymbol{\pi}_{n}(u) \mathbf{A}_{1}(u) N(u, t+k) d u \\
& =\int_{t-1}^{t} \boldsymbol{\pi}_{n}(u) \mathbf{A}_{1}(u) \hat{N}(u, t) d u,
\end{aligned}
$$

where $\hat{N}(s, t)=\sum_{k=0}^{\infty} N(s, t+k)$. The matrix function $N(s, t)$ is such that $[N(s, t)]_{i j}(1 \leq i, j \leq m)$ records the probability the process is in phase $j$ of $\ell(n)$, given the process was in phase $i$ of $\ell(n)$ at time $s$ without entering $\ell(n-1)$ during 
$(s, t)$. This probability is independent of $n \geq 1 . \sum_{k=0}^{\infty} N(u, t+k)$ is equal to the expected number of periods the process is in $\ell(n)$ at time $t$ within the period, given that it started in $\ell(n)$ at time $s$ without having entered $\ell(n-1)$ since that time.

Proof The first equality follows from the fact that if an asymptotic periodic probability vector exists, the associated random walk process will have a negative drift. If the process reaches $\ell(n+1)$, then there is a last time when the process was in $\ell(n)$. For $n>0$, the probability of not returning to $\ell(n)$ during $(s, t)$ is independent of level and is given by $N(s, t)$. The integrand gives the probability of being in $\ell(n)$, then at the time instant $u$ making a transition to $\ell(n+1)$, not returning to $\ell(n)$ during $(u, t+k)$, and being in $\ell(n+1)$ at times $u$ and $t+k$. During $(u, t+k)$ the process may visit levels greater than $\ell(n+1)$, but it does not visit $\ell(k)$ for $k \leq n$. The second equality is proved algebraically in Theorem 7 in the Appendix.

We may also arrive at this result directly. If the process is in $\ell(n+1)$ at time $t$, then there is a last time, $u$, that it was in $\ell(n) . u$ may have been in the same period as $t$ or it may have been in any previous period $k$. We use a law of total probability argument and sum over all periods $k$ and integrate over all times $u$ within the period.

Consider a class of functions, $\mathcal{R}$, such that if $F(s, t)$ is in $\mathcal{R}$ then

1. $F(s, t)=F(s+k, t+k)$ for all $k \in \mathbf{Z}$.

2. If $s>t, F(s, t)=0$.

3. For any function, $F \in \mathcal{R}$, the functions defined by $\lim _{n \rightarrow \infty} \sum_{k=0}^{n} F(s, t+k)=$ $\hat{F}(s, t)$ converge.

Note that this implies $\lim _{k \rightarrow \infty} F(s, t+k)=0$. Let $\mathbf{A}(t)$ be a periodic function with period 1, so that $\mathbf{A}(t)=\mathbf{A}(t+k)$ for $k \in \mathbf{Z}$. In Theorem 7 in the Appendix, we prove several identities related to these $\mathcal{R}$ functions.

Corollary 1 Assume that the Markov process is irreducible. If an asymptotic periodic distribution exists, then it satisfies the relation

$$
\begin{aligned}
\boldsymbol{\pi}_{n+j}(t) & =\lim _{k \rightarrow \infty} \int_{s}^{t+k} \pi_{n}(u) \mathbf{R}^{(j)}(u, t+k) d u \\
& =\int_{t-1}^{t} \pi_{n}(u) \hat{\mathbf{R}}^{(j)}(u, t) d u
\end{aligned}
$$

where $\mathbf{R}^{(j)}(s, t)$ is given by

$$
\mathbf{R}^{(j)}(s, t)=\int_{s}^{t} \mathbf{R}^{(j-1)}(s, u) \mathbf{R}(u, t) d u=\int_{s}^{t} \mathbf{R}(s, u) \mathbf{R}^{(j-1)}(u, t) d u
$$

with

$$
\begin{aligned}
& \mathbf{R}^{(1)}(s, t)=\mathbf{R}(s, t)=\mathbf{A}_{1}(s) \mathbf{N}(s, t), \\
& \text { and } \hat{\mathbf{R}}^{(j)}(u, t)=\sum_{k=0}^{\infty} \mathbf{R}^{(j)}(u, t+k) \text {. }
\end{aligned}
$$


Using a straightforward generalization of Theorem 1, we may express the matrix functions $\mathbf{R}^{(j)}(s, t)$ in terms of the generalized random walk transition probabilities:

$$
\mathbf{R}^{(j)}(s, t)=\mathbf{A}_{1}(s)\left(\phi_{j-1}(s, t)-\phi_{-1} \star \phi_{0}^{\star(-1)} \star \phi_{j}(s, t)\right) .
$$

\section{Corollary 2}

$$
\hat{\mathbf{R}}^{(j)}(s, t)=\mathbf{A}_{1}(s)\left(\hat{\phi}_{j-1}(s, t)-\int_{t-1}^{t} \hat{f}_{-1}(s, u) \hat{\phi}_{j}(u, t) d u\right)
$$

where $f_{-1}(s, t)$ is the solution to the Volterra equation of the second kind given in (3).

We may evaluate these matrix functions using the identities in the Appendix. In particular, identity 3 in Theorem 7 provides a means to compute $\hat{f}_{-1}(s, t)$. Given $\hat{f}_{-1}(s, t)$ and the $\hat{\phi}_{n}(s, t)$, we may compute $\hat{\mathbf{R}}^{(n)}(s, t)$.

In Sect. 6 we discuss the use of the normal distribution in approximating the $\phi_{n}(s, t)$.

\section{$3 \mathbf{R}$ and $\mathbf{G}$ matrix functions}

The matrices $\mathbf{R}$ and $\mathbf{G}$ of matrix analytic methods [18] have analogues in the form of matrix functions in the case of QBDs with time-varying rates.

The matrix functions $\mathbf{R}$ and $\mathbf{G}$ satisfy the following equations:

$$
\begin{aligned}
\mathbf{R}(s, t) & =\mathbf{A}_{1}(s) \mathbf{N}(s, t), \\
\mathbf{G}(s, t) & =\mathbf{N}(s, t) \mathbf{A}_{-1}(t),
\end{aligned}
$$

and

Theorem $\mathbf{3}$ (R and $\mathbf{G})$

$$
\begin{aligned}
& \mathbf{R}(s, t)=\mathbf{A}_{1}(s) \mathbf{E}_{0}(s, t)+\int_{s}^{t} \mathbf{R}(s, u) \int_{s}^{u} \mathbf{R}(u, v) \mathbf{A}_{-1}(v) \mathbf{E}_{0}(v, t) d v d u, \\
& \mathbf{G}(s, t)=\mathbf{E}_{0}(s, t) \mathbf{A}_{-1}(t)+\int_{s}^{t} \int_{s}^{u} \mathbf{E}_{0}(s, v) \mathbf{A}_{1}(v) \mathbf{G}(v, u) d v \mathbf{G}(u, t) d u,
\end{aligned}
$$

where $\mathbf{E}_{0}(s, t)$ is the evolution operator corresponding to $\mathbf{A}_{0}(t)$. We also have

$$
\begin{aligned}
\mathbf{R}(s, t)= & \delta_{s=t} \mathbf{A}_{1}(s)+\int_{s}^{t} \mathbf{R}(s, u) \mathbf{A}_{0}(u) d u \\
& +\int_{s}^{t} \mathbf{R}(s, u) \int_{s}^{u} \mathbf{R}(u, v) \mathbf{A}_{-1}(v) d v d u, \\
\mathbf{G}(s, t)= & \delta_{s=t} \mathbf{A}_{-1}(t)+\int_{s}^{t} \mathbf{A}_{0}(u) \mathbf{G}(u, t) d u \\
& +\int_{s}^{t} \int_{s}^{u} \mathbf{A}_{1}(v) \mathbf{G}(v, u) d v \mathbf{G}(u, t) d u,
\end{aligned}
$$

where $\delta_{s=t}$ is one if $s=t$, and zero otherwise. 
Proof To prove (5), note that since $\mathbf{R}(s, t)=\mathbf{A}_{1}(s) \mathbf{N}(s, t)$, if we can show

$$
\mathbf{N}(s, t)=\mathbf{E}_{0}(s, t)+\int_{s}^{t} \mathbf{N}(s, u) \int_{s}^{u} \mathbf{A}_{1}(u) \mathbf{N}(u, v) \mathbf{A}_{-1}(v) \mathbf{E}_{0}(v, t) d v d u,
$$

we are done. Recall the probabilistic interpretation of $\mathbf{N}(s, t)$ as a matrix of transition probabilities for which transitions to any level lower than the initial level are taboo. If the process is in $\ell(n)$ at both time $s$ and time $t$ and has not entered $\ell(n-1)$ during the time interval $(s, t)$, then one of two events occurred: (1) no transition outside of $\ell(n)$ occurred, or (2) some transitions occurred and the last transition was a transition from $\ell(n+1)$ to $\ell(n)$. The $(i, j)$ component of (9) gives the probability of a transition from phase $i$ to phase $j$. The probability of the first event is given by the first term on the right-hand side, and the second event is given in the second term. Equation (6) may be proved in a similar manner.

We may also prove these equations algebraically by expressing $\mathbf{N}(s, t)$ in terms of $\phi_{n}(s, t)$ and considering $\frac{\partial}{\partial t}\left[\mathbf{N}(s, t) \mathbf{E}_{0}(t, u)\right]$. The details are omitted.

Equation (7) is obtained by differentiating $\mathbf{R}(s, t)$ with respect to $t$ (with $\mathbf{R}(s, t)$ expressed in terms of $\phi_{n}(s, t)$ as in (4)) and then integrating the result.

Equation (8) is obtained by differentiating $\mathbf{G}(s, t)$ with respect to $s$ (with $\mathbf{G}(s, t)$ expressed in terms of $\left.\phi_{n}(s, t)\right)$ and then integrating the result.

Corollary $\mathbf{3}$ ( $\hat{\mathbf{R}}$ and $\hat{\mathbf{G}})$ The matrix functions $\hat{\mathbf{R}}$ and $\hat{\mathbf{G}}$ satisfy similar equations:

$$
\begin{aligned}
& \hat{\mathbf{R}}(s, t)=\mathbf{A}_{1}(s) \hat{\mathbf{N}}(s, t), \\
& \hat{\mathbf{G}}(s, t)=\hat{\mathbf{N}}(s, t) \mathbf{A}_{-1}(t),
\end{aligned}
$$

and

$$
\begin{aligned}
& \hat{\mathbf{R}}(s, t)=\mathbf{A}_{1}(s) \hat{\mathbf{E}}_{0}(s, t)+\int_{t-1}^{t} \hat{\mathbf{R}}(s, u) \int_{u-1}^{u} \hat{\mathbf{R}}(u, v) \mathbf{A}_{-1}(v) \hat{\mathbf{E}}_{0}(v, t) d v d u, \\
& \hat{\mathbf{G}}(s, t)=\hat{\mathbf{E}}_{0}(s, t) \mathbf{A}_{-1}(t)+\int_{t-1}^{t} \int_{u-1}^{u} \hat{\mathbf{E}}_{0}(s, v) \mathbf{A}_{1}(v) \hat{\mathbf{G}}(v, u) d v \hat{\mathbf{G}}(u, t) d u,
\end{aligned}
$$

where $\hat{\mathbf{E}}_{0}(s, t)=\sum_{k=0}^{\infty} \mathbf{E}_{0}(s, t+k)$ and $\mathbf{E}_{0}(s, t)$ is the solution of the evolution equation given by $\frac{\partial}{\partial t} \mathbf{E}_{0}(s, t)=\mathbf{E}_{0}(s, t) \mathbf{A}_{0}(t)$.

Proof The proof follows by applying the identities in Theorem 7 of the Appendix, to Theorem 3.

Remark 1 The matrix function $\hat{\mathbf{E}}_{0}(s, t)$ is analogous to $\left(\mathbf{I}-\mathbf{A}_{0}\right)^{-1}$ in matrix analytic methods. The integral equations given in the corollary when discretized become matrix equations for $R$ and $G$ which may be solved numerically using algorithms from matrix analytic methods.

The matrix function $\tilde{\mathbf{G}}(s)$ is defined as follows:

$$
\tilde{\mathbf{G}}_{i j}(s)=P\left\{\tau<\infty \text { and } X_{\tau}=(n-1, j) \mid X_{s}=(n, i)\right\} ;
$$


the matrix $\tilde{\mathbf{G}}(s)$ records the probability, starting from $\ell(n)$ at time $s$ of visiting $\ell(n-1)$ in a finite time. The function $\tilde{\mathbf{G}}(s)$ is important in the proof of Theorem 6 in Sect. 6. We may compute $\tilde{\mathbf{G}}(s)$ using the following integral in $\mathbf{G}(s, t)$ :

$$
\tilde{\mathbf{G}}(s)=\int_{s}^{\infty} \mathbf{G}(s, \tau) d \tau=\int_{t-1}^{t} \hat{\mathbf{G}}(s, \tau) d \tau .
$$

The matrix $\mathbf{G}(s, \tau)$ has the following probabilistic interpretation. During the time interval $(s, \tau)$ transitions occur governed by the block tri-diagonal infinitesimal generator

$$
\left[\begin{array}{ccccc}
\ddots & \ddots & 0 & 0 & \cdots \\
\mathbf{A}_{-1}(t) & \mathbf{A}_{0}(t) & \mathbf{A}_{1}(t) & 0 & \ldots \\
0 & \mathbf{A}_{-1}(t) & \mathbf{A}_{0}(t) & \mathbf{A}_{1}(t) & \cdots \\
0 & 0 & \mathbf{A}_{-1}(t) & \mathbf{A}_{0}(t) & \cdots \\
\vdots & \vdots & \vdots & \vdots & \ddots
\end{array}\right] .
$$

The process is in level $\ell(n)$ at time $s$ and again (or still) in $\ell(n)$ at time $\tau_{-}$, and at the instant $\tau$, a transition occurs to level $\ell(n-1)$. The integral of this quantity then gives the probability that at some time $\tau$ a transition occurs to $\ell(n-1)$. By integrating over all $\tau$, we get the probability that eventually there is a transition to $\ell(n-1)$. The second equality in (12) follows from identity 1 in Theorem 7.

\section{Boundary distribution}

In order to more completely specify the stationary distribution, one needs to determine the subvector $\pi_{0}(t)$. The subvector $\pi_{0}(t)$ for a positive recurrent QBD will satisfy the following integral equation:

$$
0=\int_{t-1}^{t} \boldsymbol{\pi}_{0}(u) \mathbf{B}(u) d u \mathbf{1}+\int_{t-1}^{t} \boldsymbol{\pi}_{1}(u) \mathbf{A}_{-1}(u) d u \mathbf{1} .
$$

The equation simply states that over the course of a single period, if the system is in its asymptotic periodic equilibrium, then the flow into $\ell(0)$ will equal the flow out.

This leads to the following theorem:

Theorem 4 (Boundary condition) A QBD with periodic rates will satisfy the following equations:

$$
0=\int_{t-1}^{t} \boldsymbol{\pi}_{0}(u)\left(\mathbf{B}(u)+\int_{u-1}^{u} \hat{\mathbf{R}}(u, v) \mathbf{A}_{-1}(v) d v\right) d u \mathbf{1}
$$

and

$$
\int_{t-1}^{t} \boldsymbol{\pi}_{0}(u) \sum_{j=0}^{\infty} \hat{\mathbf{R}}^{(j)}(u, t) d u \mathbf{1}=1 .
$$


Proof The first term in the first equation is the first term in (13). For the second term, we apply Corollary 1 and change the order of integration. The second equation is equivalent to $\sum_{j=0}^{\infty} \boldsymbol{\pi}_{j}(t) \mathbf{1}=1$.

We note that

$$
\hat{\mathbf{S}}(s, t)=\sum_{j=1}^{\infty} \hat{\mathbf{R}}^{(j)}(s, t) d u
$$

is the solution of the following Volterra equation of the second kind:

$$
\hat{\mathbf{S}}(s, t)=\hat{\mathbf{R}}(s, t)+\int_{t-1}^{t} \hat{\mathbf{R}}(s, u) \hat{\mathbf{S}}(u, t) d u,
$$

so given $\hat{\mathbf{R}}(s, t)$, we can compute $\hat{\mathbf{S}}(s, t)$ and then normalize our subvector $\boldsymbol{\pi}_{0}(t)$ using

$$
\boldsymbol{\pi}_{0}(t) \mathbf{1}+\int_{t-1}^{t} \boldsymbol{\pi}_{0}(u) \hat{\mathbf{S}}(u, t) d u \mathbf{1}=1 .
$$

The function $\hat{\mathbf{S}}(s, t)$ is analogous to $\mathbf{R}(\mathbf{I}-\mathbf{R})^{-1}$ of matrix analytic methods.

When $\mathbf{B}(t)=\mathbf{A}_{-1}(t)+\mathbf{A}_{0}(t)$, we may adapt Lemma 6.3.2 from [18] to the case of QBDs with time-varying parameters, and we have

Lemma 2 Assume that the $Q B D$ is irreducible, that it is positive recurrent, and that $\mathbf{B}(t)=\mathbf{A}_{-1}(t)+\mathbf{A}_{0}(t)$. Then

$$
\boldsymbol{\pi}_{n}(t)=\int_{t-1}^{t} \boldsymbol{\alpha}(u)\left(\hat{\mathbf{R}}^{(n)}(u, t)-\hat{\mathbf{R}}^{(n+1)}(u, t)\right) d u
$$

where $\boldsymbol{\alpha}(u)$ is the asymptotic periodic solution of the finite state Markov chain with generator $\mathbf{A}(t)$.

The proof is a straightforward generalization of the results in [18] and the details are omitted.

\section{Stability condition}

Theorem 5 (Stability condition) If the $Q B D$ is irreducible, if $m$ is finite, and if the infinitesimal generator $\mathbf{A}_{-1}(t)+\mathbf{A}_{0}(t)+\mathbf{A}_{1}(t)=\mathbf{A}(t)$ is irreducible, then the $Q B D$ is positive recurrent if and only if

$$
\bar{\mu}=\int_{0}^{T} \boldsymbol{\alpha}(u)\left(\mathbf{A}_{1}(u)-\mathbf{A}_{-1}(u)\right) d u \mathbf{1}<0,
$$

where $\boldsymbol{\alpha}(u)$ is the periodic asymptotic probability vector of $\mathbf{A}(t)$. The $Q B D$ is null recurrent if $\bar{\mu}=0$, and it is transient if $\bar{\mu}>0$. 
Proof The proof is based on the approach taken in [18, Theorem 7.2.3, p. 157]. Latouche and Ramaswami consider a discrete time QBD and associate with it a random walk. We consider a continuous time periodic QBD, and associate with it a continuous time generalized random walk [25], and then we associate a family of embedded discrete time random walks for each time $s$ within the period.

The generalized random walk $\left\{\left(\eta_{t}, \zeta_{t}, t-\lfloor t\rfloor\right), t \geq 0\right\}$ is the process governed by the Chapman-Kolmogorov forward equations:

$$
\dot{\mathbf{p}}_{n}(t)=\mathbf{p}_{n-1}(t) \mathbf{A}_{1}(t)+\mathbf{p}_{n}(t) \mathbf{A}_{0}(t)+\mathbf{p}_{n+1}(t) \mathbf{A}_{-1}(t), \quad n \in \mathbb{Z},
$$

with state space $\mathbb{Z} \times\{1, \ldots, m\} \times[0,1)$ with transition rates depending on the phase, $\zeta_{t}$, and the time within the period, $t-\lfloor t\rfloor$, but not on the level $\eta_{t}$. The steps of the random walk are the levels but, for the generalized random walk, we also associate a phase with each step. Observe that the QBD and the generalized random walk have the same behavior until the first visit to $\ell(0)$.

We define $\epsilon_{n}^{(s)}=\eta_{s+n}-\eta_{s+n-1}$ and consider the family of embedded discrete Markov chains, $\left\{\left(\epsilon_{n}^{(s)}, \zeta_{s+n}\right), n \in \mathbb{N}\right\}, s \in[0,1)$, with state space $\mathbb{Z} \times\{1, \ldots, m\}$, obtained by observing the generalized random walk at the end of each period. Transition probabilities

$$
\left[\phi_{k}(s, s+1)\right]_{i j}=P\left\{\epsilon_{n+1}^{(s)}=k, \zeta_{s+n+1}=j \mid \epsilon_{n}^{(s)}, \zeta_{s+n}=i\right\}, \quad k \in \mathbb{Z},
$$

depend only on the second coordinate. Examples of the matrix functions $\phi_{k}(s, t)$ are given in Sect. 2. Their more complete description is available in [25]. Defining $L_{n+s}=L_{s}+\sum_{1 \leq v \leq n} \epsilon_{v}^{(s)}$, we have $\left\{L_{s+n}, \zeta_{s+n}\right\}, n \in \mathbb{N}$, on the state space $\mathbb{Z} \times\{1, \ldots, m\}$ and $L_{s+n}$ constitutes a random walk on the integers with increments $\left\{\epsilon_{n}^{(s)}\right\}$ to $L_{s+n}$ controlled by the Markov chain $\left\{\zeta_{s+n}\right\}$. Let $\gamma=\inf \left\{n \geq 1: L_{s+n} \leq 0\right\}$ be the first passage time of the discrete Markov chain $\left\{L_{s+n}, \zeta_{s+n}\right\}$ to the negative half-line. Note that the generalized random walk may have reached the negative halfline some time earlier than the discretized process.

If we choose $\zeta_{s}$ according to the stationary distribution $\boldsymbol{\alpha}(s)$, then

Lemma 3 (Mean drift) The process $\epsilon_{n}$ is stationary with

$$
E\left[\epsilon_{n}^{(s)}\right]=\bar{\mu}=\int_{0}^{T} \boldsymbol{\alpha}(u)\left(\mathbf{A}_{1}(u)-\mathbf{A}_{-1}(u)\right) d u \mathbf{1} .
$$

Proof The expectation of the level for the generalized random walk is given by differentiating the generating function $\Phi_{y}(s, s+T)$ (for rates periodic with period $T$ ) with respect to $y$, setting $y=1$, pre-multiplying the resulting matrix by $\boldsymbol{\alpha}(s)$, the initial distribution vector, and post-multiplying by the column vector $\mathbf{1}$. This yields (see [25] for details):

$$
\boldsymbol{\alpha}(s) \int_{s}^{s+T} \mathbf{V}(s, u)\left(\mathbf{A}_{1}(u)-\mathbf{A}_{-1}(u)\right) \mathbf{V}(u, s+T) d u \mathbf{1}
$$

where $\mathbf{V}(s, t)$ is an evolution operator such that $\frac{\partial}{\partial t} \mathbf{V}(s, t)=\mathbf{V}(s, t) \mathbf{A}(t), \mathbf{V}(t, t)=\mathbf{I}$. $\mathbf{V}(s, t)$ is a probability transition matrix for the generator $\mathbf{A}(t)=\mathbf{A}_{-1}(t)+\mathbf{A}_{0}(t)+$ 
$\mathbf{A}_{1}(t)$, so $\boldsymbol{\alpha}(s) \mathbf{V}(s, u)=\boldsymbol{\alpha}(u)$ and $\mathbf{V}(u, s+T) \mathbf{1}=\mathbf{1}$. Hence the mean drift is

$$
\begin{aligned}
& \boldsymbol{\alpha}(s) \int_{s}^{s+T} \mathbf{V}(s, u)\left(\mathbf{A}_{1}(u)-\mathbf{A}_{-1}(u)\right) \mathbf{V}(u, s+T) d u \mathbf{1} \\
& =\int_{s}^{s+T} \boldsymbol{\alpha}(u)\left(\mathbf{A}_{1}(u)-\mathbf{A}_{-1}(u)\right) d u \mathbf{1} \\
& =\int_{0}^{T} \boldsymbol{\alpha}(u)\left(\mathbf{A}_{1}(u)-\mathbf{A}_{-1}(u)\right) d u \mathbf{1}
\end{aligned}
$$

We consider the case when the period $T=1$.

The process $\left\{L_{n+s}\right\}$ has stationary increments. The Markov chain $\left\{\left(\epsilon_{n}^{(s)}, \zeta_{s+n}\right)\right\}$ has a stationary distribution, $\boldsymbol{\beta}(s)$, with the $j$ th component of subvector $\boldsymbol{\beta}_{k}(s)=$ $\boldsymbol{\alpha}(s) \phi_{k}(s, s+1)$ corresponding to the probability that $\epsilon_{n}^{(s)}=k$ and $\zeta_{s+n}=j$.

The states of the Markov chain $\left\{\left(\epsilon_{n}^{(s)}, \zeta_{s+n}\right)\right\}$ are positive recurrent, so they form an ergodic process and $\left\{\epsilon_{n}^{(s)}\right\}$ is itself ergodic. We then apply the strong law of large numbers for ergodic processes and conclude that $\lim _{n \rightarrow \infty} L_{s+n} /(s+n)=\bar{\mu}$ a.s. Since the limit holds for each $s$, we conclude that $\lim _{t \rightarrow \infty} L_{t} / t=\bar{\mu}$, for $t>0$.

If $\bar{\mu}>0$, this implies that $\lim _{t \rightarrow \infty} L_{t}=+\infty, P[\gamma<\infty]<1$, and therefore $\mathbf{G}(s)$ is substochastic, so there must be at least one index $j$ such that $(\mathbf{G}(s) \mathbf{1})_{j}<1$, so that starting from $(1, j)$, there is a strictly positive probability of never reaching $\ell(0)$. Hence, the QBD is transient.

If $\bar{\mu}<0$, this implies that $\lim _{t \rightarrow \infty} L_{t}=-\infty$, a.s., so $\gamma$ is finite a.s., and therefore $\mathbf{G}(s)$ is stochastic. To prove that $E[\gamma]$ is finite, we define the sequence of renewal epochs $k_{0}=s, k_{i+1}=\inf \left\{n+s>k_{i}: \epsilon_{n}^{(s)}=\epsilon_{0}^{(s)}, \zeta_{n+s}=\zeta_{s}\right\}$, when the process $\left\{\left(\epsilon_{n}^{(s)}, \zeta_{s+n}\right)\right\}$ returns to its initial state. Furthermore, we define $\mathcal{L}_{i}=L_{k_{i}}$ as the state of the process $\left(L_{s+n}, \zeta_{s+n}\right)$ at the $i$ th return to $\left(\epsilon_{0}^{(s)}, \zeta_{s}\right)$. We have suppressed the dependence of the $\mathcal{L}_{i}$ on $s$ in the notation.

By the strong Markov property, the increments $\left\{\mathcal{L}_{i+1}-\mathcal{L}_{i}\right\}$ are i.i.d. and $\left\{\mathcal{L}_{i}\right\}$ is a regular random walk with independent increments. Since the difference $\mathcal{L}_{i+1}-$ $\mathcal{L}_{i}=\sum_{k_{i}+1 \leq n \leq k_{i+1}} \epsilon_{n}^{(s)}$, we find that $E\left[\mathcal{L}_{i+1}-\mathcal{L}_{i}\right]=\bar{\mu} E\left[k_{1}\right]$ for all initial states and is strictly negative. Thus, the random walk $\left\{\mathcal{L}_{i}\right\}$ is transient, $\lim _{i \rightarrow \infty} \mathcal{L}_{i}=-\infty$, and $E[v]<\infty$, where $v=\inf \left\{i>0: \mathcal{L}_{i}<0\right\}$. Since $\gamma \leq k_{\nu}$, this proves $E[\gamma] \leq$ $E\left[k_{1}\right] E[\nu]$ is finite when $\bar{\mu}<0$. This result holds for each $s \in[0,1)$. Hence we conclude that for $\bar{\mu}<0$, the QBD is positive recurrent.

The proof for $\bar{\mu}=0$ proceeds as in Latouche and Ramaswami [18] with the random walk $\left\{\mathcal{L}_{i}\right\}$ replacing the one used in their proof.

\section{Conclusion}

In this section we provide some initial ideas as to how these formulas might be used for computation.

The random walk transition probabilities, $\phi_{k}(s, t)$, and expected number of visits to level $k$ at time $t$ within the period, $\hat{\phi}_{k}(s, t)$, are important in many of the formu- 


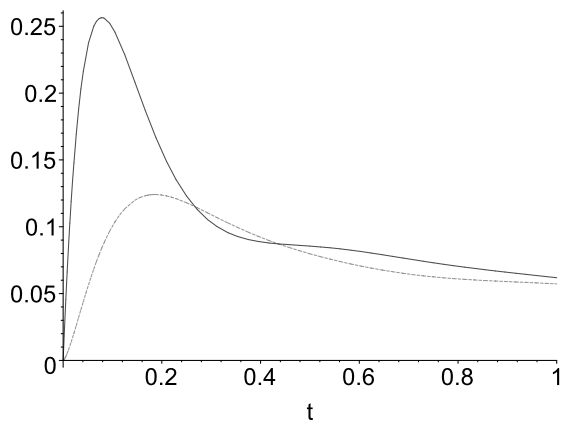

(a) $t=0$ to 1

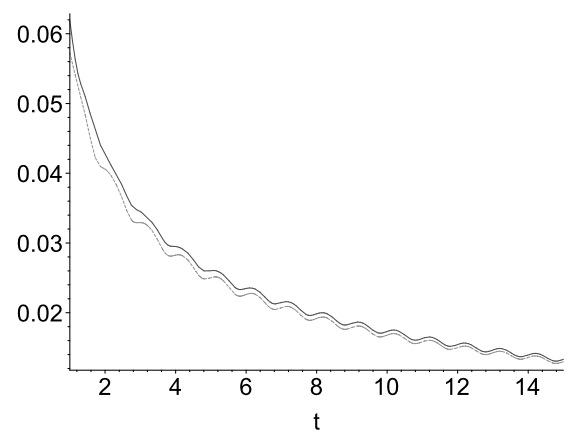

(b) $t=1$ to 15

Fig. 1 Normal density approximation of $\left[\phi_{0}(0, t)\right]_{1,1}$ for the $M / E_{4} / 1$ queue with $\lambda(t)=2+2 \cos (2 \pi t)$ and $v(t)=9$. The thicker, dashed line is the approximation

las given in the preceding sections. We can use the following result to approximate $\phi_{k}(s, t)$ with a normal density:

Theorem 6 Consider a generalized random walk process with mean drift during the time interval $[s, t+n)$ equal to $\mu(s, t+n)$ and variance $\sigma^{2}(s, t+n)$. Let $z_{n k}=$ $\frac{k-\mu(s, t+n)}{\sigma(s, t+n)}$. The relation

$$
\sigma(s, t+n) p_{k}(t)-\frac{1}{\sqrt{2 \pi}} e^{-\frac{z_{n k}^{2}}{2}} \rightarrow 0
$$

as $n \rightarrow \infty$ holds uniformly with respect to $k \in \mathbb{Z}$.

Figure 1 illustrates this convergence for the $M / E_{4} / 1$ queue with $\lambda(t)=2+$ $2 \cos (2 \pi t)$ and $v(t)=9$. The generalized random walk associated with the $M / E_{4} / 1$ queue, has $\mu(s, t)=\int_{s}^{t}\left(\lambda(u)-\frac{v(u)}{4}\right) d u$ and $\sigma^{2}(s, t)=\frac{5}{32}+\frac{5}{16} \int_{s}^{t} v(u) d u-$ $\frac{1}{32} e^{-2 \int_{s}^{t} v(u) d u}-\frac{1}{8} e^{-\int_{s}^{t} v(u) d u} \cos \left(\int_{s}^{t} v(u) d u\right)$. The normal approximation is shown by the dashed line. Note that the approximation is very good for larger values of $t$ but not as good for $t$ close to zero. When we increase the rates, we get more rapid convergence as can be seen in Fig. 2.

The proof of this result is based on the proof of a local limit theorem for the sum of $n$ independent, identically distributed lattice random variables as presented in Gnedenko and Kolmogorov [9, Sect. 49]. The details will be presented in a subsequent paper focusing on computation and estimation. A key point in the proof is that the variance of the levels for the generalized random walk is asymptotically linear.

We consider the $M_{t} / M_{t} / 1$ queue.

There are several possible approaches that we can use to compute the idle probability for the $M_{t} / M_{t} / 1$ queue. We may apply Theorem 3 in [25] which provides the following formula for $\pi_{0}(t)$ :

$$
\pi_{0}(t)=\int_{t-1}^{t} \pi_{0}(\nu) \mu(\nu)\left(\hat{\phi}_{0}(\nu, t)-\hat{\phi}_{1}(\nu, t)\right) d \nu .
$$




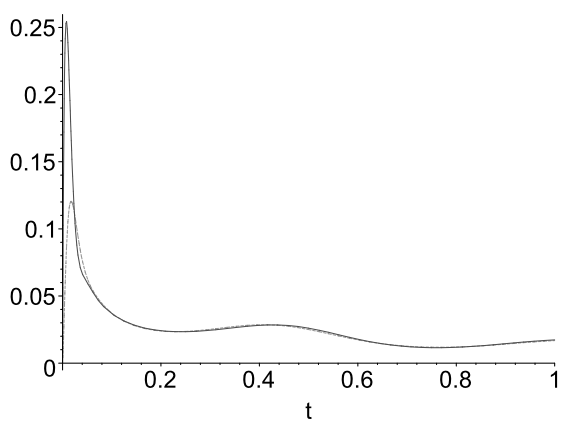

(a) $t=0$ to 1

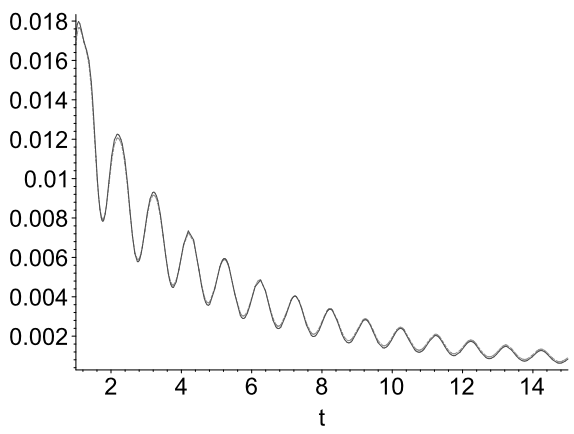

(b) $t=1$ to 15

Fig. 2 Normal density approximation of $\left[\phi_{0}(0, t)\right]_{1,1}$ for the $M / E_{4} / 1$ queue with $\lambda(t)=20+20 \cos (2 \pi t)$ and $v(t)=90$. The thicker, dashed line is the approximation

We truncate the infinite series $\hat{\phi}_{0}(v, t)$ and $\hat{\phi}_{1}(v, t)$. We compute the first two terms of the series directly and use the normal density approximation given by Theorem 6 for the next several terms $\phi_{0}(v, t+k)$ and $\phi_{1}(v, t+k)$ of the series. The resulting numerical estimate for $\pi_{0}(t)$ must then be normalized. We can do this using the relation $\bar{\mu}-\bar{\lambda}=\int_{t-1}^{t} \pi_{0}(v) \mu(v) d v$ where $\bar{\mu}=\int_{0}^{1} \mu(u) d u$ and $\bar{\lambda}=\int_{0}^{1} \lambda(u) d u$ are the average number of steps to the left and steps to the right, respectively, during a period for the generalized random walk corresponding to the $M_{t} / M_{t} / 1$ queue.

An alternative approach is to compute the $\hat{\mathbf{R}}(s, t)$ function for $s, t \in[0,1]$ using the formula (10) given in Corollary 3 to Theorem 3. After discretization for numerical integration, this formula is simply a matrix quadratic equation, and any algorithm that solves matrix quadratic equations may be employed. We apply Lemma 2 to estimate $\pi_{0}(t)$.

A third alternative is to compute the $\hat{\mathbf{R}}(s, t)$ function for $s, t \in[0,1]$ using formula (4).

Given $\pi_{0}(t)$, we compute the expected number in the queue using the formula [25, p. 191]:

$$
E[X(t)]=\frac{\bar{\lambda}}{\bar{\mu}-\bar{\lambda}}+\frac{\bar{\mu}-\bar{\lambda}}{2}+\frac{\int_{t-1}^{t} \mu(v) \pi_{0}(v) \int_{v}^{t}(\lambda(\xi)-\mu(\xi)) d \xi d v}{\bar{\mu}-\bar{\lambda}}
$$

Using any of these approaches for the $M_{t} / M_{t} / 1$ example given in [28] with $\lambda(t)=$ $1+\sin (2 \pi t)$ and $\mu(t)=4+2 \cos (2 \pi t)$ with a mesh of $n=500$, we obtain the same result as Zeifman et al. for the average expected number in the queue over the period to three decimal places of accuracy. The idle probability and expected number in the queue are shown in Fig. 3.

This paper places continuous time quasi-birth-and-death processes with timevarying periodic rates in the framework of matrix analytic methods. In this section, we have provided some indications as to how these formulas might be used for computation. Future work will explore computation and numerical considerations more fully. 


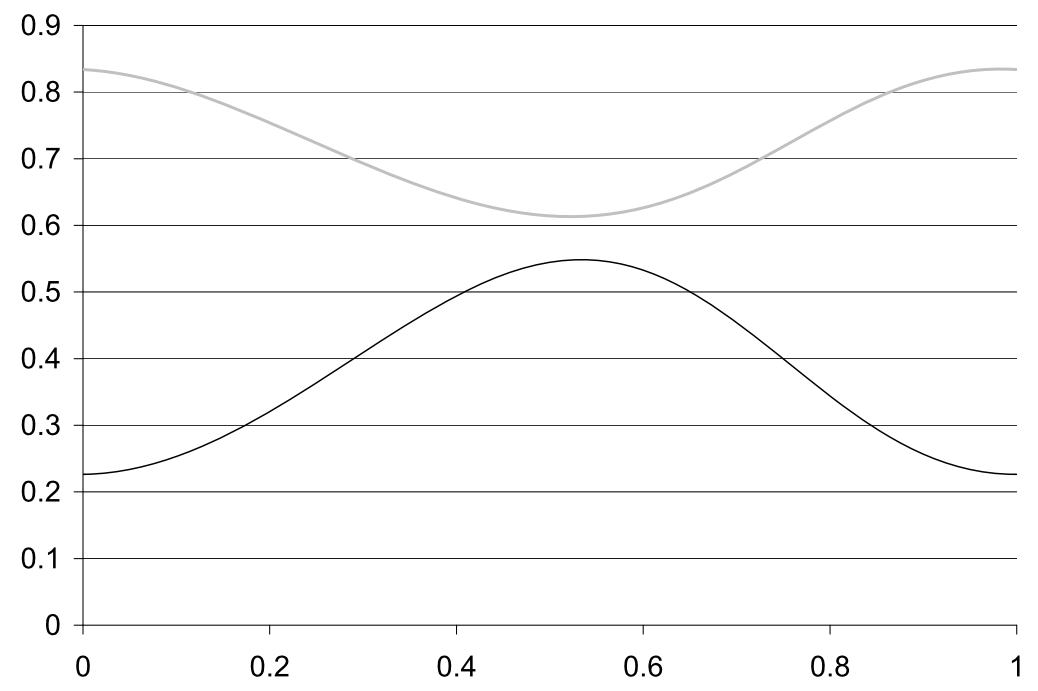

Fig. $3 M_{t} / M_{t} / 1$ queue with $\lambda(t)=1+\sin (2 \pi t)$ and $\mu(t)=4+2 \cos (2 \pi t)$. The idle probability is shown in gray, and the expected number in the queue is shown in black

\section{Appendix: Class $\mathcal{R}$ function integral identities}

Let $F, G$ and $H$ be functions in $\mathcal{R}$, and $t-s<1$, then

Theorem 7 (Identities) The following identities are valid:

1.

$$
\lim _{n \rightarrow \infty} \int_{s}^{t+n} \mathbf{A}(u) F(u, t+n) d u=\int_{t-1}^{t} \mathbf{A}(u) \hat{F}(u, t) d u .
$$

2. If $F(s, t)=\int_{s}^{t} G(s, u) H(u, t) d u$, then

$$
\hat{F}(s, t)=\int_{t-1}^{t} \hat{G}(s, u) \hat{H}(u, t) d u .
$$

3. If

$$
F(s, t)=G(s, t)+\int_{s}^{t} F(s, u) H(u, t) d u
$$

then

$$
\hat{F}(s, t)=\hat{G}(s, t)+\int_{t-1}^{t} \hat{F}(s, u) \hat{H}(u, t) d u .
$$

4. If $F(s, t)=\int_{s}^{t} G(s, u) H(u, t) d u$, then

$$
\lim _{n \rightarrow \infty} \int_{s}^{t+n} \mathbf{A}(u) F(u, t+n) d u=\int_{t-1}^{t} \mathbf{A}(u) \int_{t-1}^{t} \hat{G}(u, v) \hat{H}(v, t) d v d u .
$$


5.

$$
\hat{F}(s+k, t)=\hat{F}(s, t)
$$

for $k=0,1,2, \ldots$

6.

$$
\hat{F}(s, t+k)=\hat{F}(s, t)-\sum_{j=0}^{k-1} F(s, t+j)
$$

for $k=0,1,2, \ldots$

Proof 1. Since $\int_{a}^{b} f(u) d u=\int_{a}^{c} f(u) d u+\int_{c}^{b} f(u) d u$,

$$
\begin{aligned}
& \lim _{n \rightarrow \infty} \int_{s}^{t+n} \mathbf{A}(u) F(u, t+n) d u \\
& \quad=\lim _{n \rightarrow \infty}\left[\int_{s}^{t} \mathbf{A}(u) F(u, t+n) d u+\int_{t}^{t+n} \mathbf{A}(u) F(u, t+n) d u\right] .
\end{aligned}
$$

Note that $\lim _{n \rightarrow \infty} F(u, t+n)=0$, so $\lim _{n \rightarrow \infty} \int_{s}^{t} \mathbf{A}(u) F(u, t+n) d u=0$ also and we have

$$
\lim _{n \rightarrow \infty} \int_{s}^{t+n} \mathbf{A}(u) F(u, t+n) d u=\lim _{n \rightarrow \infty} \int_{t}^{t+n} \mathbf{A}(u) F(u, t+n) d u .
$$

We apply $\int_{a}^{b} f(u) d u=\int_{a}^{c} f(u) d u+\int_{c}^{b} f(u) d u$ repeatedly and obtain

$$
\lim _{n \rightarrow \infty} \int_{s}^{t+n} \mathbf{A}(u) F(u, t+n) d u=\lim _{n \rightarrow \infty} \sum_{j=1}^{n} \int_{t+j-1}^{t+j} \mathbf{A}(u) F(u, t+n) d u .
$$

Changing variables yields

$$
\lim _{n \rightarrow \infty} \int_{s}^{t+n} \mathbf{A}(u) F(u, t+n) d u=\lim _{n \rightarrow \infty} \sum_{j=1}^{n} \int_{t-1}^{t} \mathbf{A}(u+j) F(u+j, t+n) d u .
$$

Since $F(\cdot, \cdot) \in \mathcal{R}$ and $\mathbf{A}(t)$ is periodic, we have

$$
\lim _{n \rightarrow \infty} \int_{s}^{t+n} \mathbf{A}(u) F(u, t+n) d u=\lim _{n \rightarrow \infty} \sum_{j=1}^{n} \int_{t-1}^{t} \mathbf{A}(u) F(u, t+n-j) d u .
$$

Let $k=n-j$,

$$
\lim _{n \rightarrow \infty} \int_{s}^{t+n} \mathbf{A}(u) F(u, t+n) d u=\lim _{n \rightarrow \infty} \sum_{k=0}^{n-1} \int_{t-1}^{t} \mathbf{A}(u) F(u, t+k) d u,
$$


interchange the order of integration and summation, and bring the limit inside the integral; then we have

$$
\lim _{n \rightarrow \infty} \int_{s}^{t+n} \mathbf{A}(u) F(u, t+n) d u=\int_{t-1}^{t} \mathbf{A}(u) \sum_{k=0}^{\infty} F(u, t+k) d u .
$$

Finally, applying the definition of $\hat{F}(\cdot, \cdot)$, we have

$$
\lim _{n \rightarrow \infty} \int_{s}^{t+n} \mathbf{A}(u) F(u, t+n) d u=\int_{t-1}^{t} \mathbf{A}(u) \hat{F}(u, t) d u .
$$

2. If $F(s, t)=\int_{s}^{t} G(s, u) H(u, t) d u$, then by definition, we have

$$
\hat{F}(s, t)=\sum_{j=0}^{\infty} \int_{s}^{t+j} G(s, u) H(u, t+j) d u .
$$

Since $\int_{a}^{b} f(u) d u=\int_{a}^{c} f(u) d u+\int_{c}^{b} f(u) d u$,

$$
\begin{aligned}
\hat{F}(s, t) & =\sum_{j=0}^{\infty} \int_{s}^{t} G(s, u) H(u, t+j) d u+\sum_{j=0}^{\infty} \int_{t}^{t+j} G(s, u) H(u, t+j) d u \\
& =\int_{s}^{t} G(s, u) \hat{H}(u, t) d u+\sum_{j=0}^{\infty} \sum_{k=1}^{j} \int_{t+k-1}^{t+k} G(s, u) H(u, t+j) d u .
\end{aligned}
$$

A change of variables yields

$$
\hat{F}(s, t)=\int_{s}^{t} G(s, u) \hat{H}(u, t) d u+\sum_{j=0}^{\infty} \sum_{k=1}^{j} \int_{t-1}^{t} G(s, u+k) H(u+k, t+j) d u .
$$

Since $H(\cdot, \cdot)$ is in $\mathcal{R}$, we may write

$$
\hat{F}(s, t)=\int_{s}^{t} G(s, u) \hat{H}(u, t) d u+\sum_{j=0}^{\infty} \sum_{k=1}^{j} \int_{t-1}^{t} G(s, u+k) H(u, t+j-k) d u .
$$

We change first the order of summation, and then the index of summation, letting $l=j-k$ to yield

$$
\begin{aligned}
\hat{F}(s, t) & =\int_{s}^{t} G(s, u) \hat{H}(u, t) d u+\sum_{k=1}^{\infty} \sum_{j=k}^{\infty} \int_{t-1}^{t} G(s, u+k) H(u, t+j-k) d u \\
& =\int_{s}^{t} G(s, u) \hat{H}(u, t) d u+\sum_{k=1}^{\infty} \sum_{l=0}^{\infty} \int_{t-1}^{t} G(s, u+k) H(u, t+l) d u .
\end{aligned}
$$


Changing the order of integration and summation, and using the definition of $\hat{G}(\cdot, \cdot)$ and $\hat{H}(\cdot, \cdot)$, gives

$$
\hat{F}(s, t)=\int_{s}^{t} G(s, u) \hat{H}(u, t) d u+\int_{t-1}^{t} \hat{G}(s, u+1) \hat{H}(u, t) d u,
$$

and finally, since $G(s, u)=0$ for $u<s$,

$$
\hat{F}(s, t)=\int_{t-1}^{t} \hat{G}(s, u) \hat{H}(u, t) d u .
$$

3. By assumption,

$$
F(s, t)=G(s, t)+\int_{s}^{t} F(s, u) H(u, t) d u,
$$

so

$$
F(s, t+j)=G(s, t+j)+\int_{s}^{t+j} F(s, u) H(u, t+j) d u .
$$

Summing from $j=0$ to infinity, we have

$$
\hat{F}(s, t)=\hat{G}(s, t)+\sum_{j=0}^{\infty} \int_{s}^{t+j} F(s, u) H(u, t+j) d u
$$

from the definition of $\hat{F}(s, t)$ and $\hat{G}(s, t)$. From part 2,

$$
\hat{F}(s, t)=\hat{G}(s, t)+\int_{t-1}^{t} \hat{F}(s, u) \hat{H}(u, t) d u .
$$

4. From part 1 of this theorem, we have

$$
\lim _{n \rightarrow \infty} \int_{s}^{t+n} \mathbf{A}(u) F(u, t+n) d u=\int_{t-1}^{t} \mathbf{A}(u) \hat{F}(u, t) d u .
$$

By definition of $\hat{F}(s, t)$,

$$
\lim _{n \rightarrow \infty} \int_{s}^{t+n} \mathbf{A}(u) F(u, t+n) d u=\int_{t-1}^{t} \mathbf{A}(u) \sum_{j=0}^{\infty} \int_{u}^{t+j} G(u, v) H(v, t+j) d v d u .
$$

Now from part 2,

$$
\begin{gathered}
\int_{t-1}^{t} \mathbf{A}(u) \sum_{j=0}^{\infty} \int_{u}^{t+j} G(u, v) H(v, t+j) d v d u \\
=\int_{t-1}^{t} \mathbf{A}(u) \int_{t-1}^{t} \hat{G}(u, v) \hat{H}(v, t) d v d u .
\end{gathered}
$$

5. For $F(s, t) \in \mathcal{R}, F(s+k, t)=0$ for $k=1,2,3, \ldots$ By assumption, $F(s, t) \in \mathcal{R}$. 6. This is direct from the definition of $\hat{F}(s, t)$. 


\section{References}

1. Alfa, A.S.: Time-inhomogeneous bulk server queue in discrete time: A transportation type problem. Oper. Res. 30, 650-658 (1982)

2. Alfa, A.S., Margolius, B.H.: Two classes of time-inhomogeneous Markov chains: analysis of the periodic case. Ann. Oper. Res. 160, 121-137 (2008)

3. Afanas'eva, L.G.: Queuing systems with cyclic control processes. Cybern. Syst. Anal. 41, 43-55 (2005)

4. Breuer, L.: The periodic BMAP/PH/c queue. Queueing Syst. 38, 67-76 (2001)

5. Daniel, J.I.: Congestion pricing and capacity of large hub airports: a bottleneck model with stochastic queues. Econometrica 63, 327-370 (1995)

6. Delcoigne, F., Proutière, A., Régnié, G.: Modeling integration of streaming and data traffic. Perform. Eval. 55, 185-209 (2004)

7. Feldman, Z., Mandelbaum, A., Massey, W.A., Whitt, W.: Staffing of time-varying queues to achieve time-stable performance. Manag. Sci. 54, 324-338 (2008)

8. Feller, W.: An Introduction to Probability Theory and Its Applications, vol. 2. Wiley, New York (1968)

9. Gnedenko, B.V., Kolmogorov, A.N.: Limit Distributions for Sums of Independent Random Variables. Addison-Wesley, Reading (1968)

10. Gnedenko, B.V., Kovalenko, I.N.: Introduction to Queueing Theory, 2nd edn. Birkhaüser, Boston (1989)

11. Green, L., Kolesar, P.: The pointwise stationary approximation for queues with nonstationary arrivals. Manag. Sci. 37, 84-97 (1991)

12. Green, L., Kolesar, P.: The lagged PSA for estimating peak congestion in multiserver Markovian queues with periodic arrival rates. Manag. Sci. 43, 80-87 (1997)

13. Harrison, J.M., Lemoine, A.J.: Limit theorems for periodic queues. J. Appl. Probab. 14, 566-576 (1977)

14. Kolmogorov, A.N.: Sur le probleme d'attente. Mat. Sb. 38, 101 (1931) (French)

15. Kolmogorov, A.N.: The waiting problem. In: Shiryayev, A.N. (ed.) Selected Works of A.N. Kolmogorov. Probability Theory and Mathematical Statistics, vol. II, pp. 109-114. Kluwer Academic, Dordrecht (1991), translated by G. Lindquist

16. Koole, G., Mandelbaum, A.: Queueing models of call centers: an introduction. Ann. Oper. Res. 113, 41-59 (2002)

17. Koopman, B.: Air-terminal queues under time dependent conditions. Oper. Res. 20, 1089-1114 (1972)

18. Latouche, G., Ramaswami, V.: Introduction to Matrix Analytic Methods in Stochastic Modeling. SIAM, Philadelphia (1999)

19. Leese, E.L., Boyd, D.W.: Numerical methods of determining the transient behavior of queues with variable rates. J. Can. Oper. Res. Soc. 4, 1-13 (1966)

20. Mandelbaum, A., Massey, W.A.: Strong approximations for time-dependent queues. Math. Oper. Res. 20, 33-64 (1995)

21. Mandelbaum, A., Massey, W.A., Reiman, M.I.: Strong approximations for Markovian service networks. Queueing Syst. 30, 149-201 (1998)

22. Margolius, B.H.: A sample path analysis of the $M_{t} / M_{t} / c$ queue. Queueing Syst. 31, 59-93 (1999)

23. Margolius, B.H.: Transient solution to the time-dependent multi-server Poisson queue. J. Appl. Prob. 42, 766-777 (2005)

24. Margolius, B.H.: Periodic solution to the time-inhomogeneous multi-server Poisson queue. Oper. Res. Lett. 35, 125-138 (2007)

25. Margolius, B.H.: Transient and periodic solution to the time-inhomogeneous Quasi-Birth Death Process. Queueing Syst. Special Issue 56, 183-194 (2007). Second Madrid Conference on Queueing Theory

26. Neuts, M.F.: Matrix-Geometric Solutions in Stochastic Models: An Algorithmic Approach. Dover, New York (1995)

27. Peterson, M.D., Bertsimas, D.J., Odoni, A.R.: Models and algorithms for transient queueing congestion at airports. Manag. Sci. 41, 1279-1295 (1995)

28. Zeifman, A., Leorato, S., Orsingher, E., Satin, Ya., Shilova, G.: Some universal limits for nonhomogeneous birth and death processes. Queueing Syst. 52, 139-151 (2006)

29. Zhang, J., Coyle, E.J.: The transient solution of time-dependent $M / M / 1$ queues. IEEE Trans. Inf. Theory 37, 1690-1696 (1991)

Post-print standardized by MSL Academic Endeavors, the imprint of the Michael Schwartz Library at Cleveland State University, 2017 